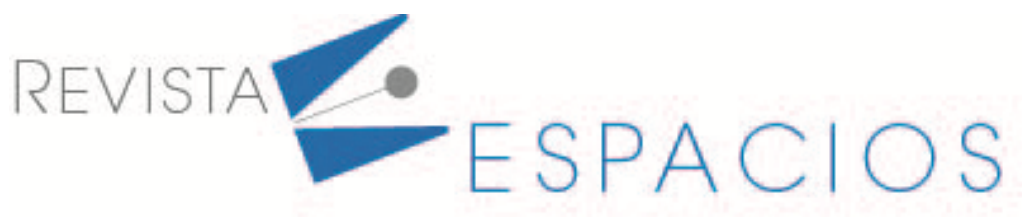

\title{
Gestión del mantenimiento para la confiabilidad operacional
}

\section{Maintenance management for operational reliability}

\author{
GASCA, Maira C. ${ }^{1}$ \\ CAMARGO, Luis L. ${ }^{2}$ \\ MEDINA, Byron ${ }^{3}$
}

\begin{abstract}
Resumen
Se propone un modelo de gestión del mantenimiento para mejorar el nivel de confiabilidad operacional en las pequeñas y medianas empresas de la industria manufacturera de la ciudad de Santa Marta, Colombia. El modelo se elaboró con base en la descripción de las variables de investigación en la población de estudio. El modelo combina prácticas y herramientas del mantenimiento centrado en confiabilidad y el mantenimiento productivo total, con el propósito de mejorar la operación y producción de las empresas.

Palabras clave: mantenimiento, confiabilidad operacional, pymes, industria manufacturera

Abstract

A maintenance management model is proposed to improve the level of operational reliability in small and medium-sized companies in the manufacturing industry in the city of Santa Marta, Colombia. The model was developed based on the description of the research variables in the study population. The model combines maintenance practices and tools focused on reliability and total productive maintenance, with the purpose of improving the operation and production of companies.

key words: maintenance, operational reliability, SMEs, manufacturing industry
\end{abstract}

\section{Introducción}

Las empresas a nivel mundial adecuan su funcionamiento interno a las exigencias del entorno, definiendo su política para aprovechar las oportunidades del contexto, de acuerdo con sus capacidades, recursos y visión. La gestión representa las estrategias y mecanismos utilizados para articular y mejorar los procesos, con el propósito de alcanzar los indicadores, metas y objetivos empresariales (González, 2010). Algunas estrategias utilizadas en las industrias para obtener el éxito son: la optimización racional de los costos de operación (Rojas, Valencia, \& Cuartas, 2017), la incorporación de tecnologías al aparato productivo (Medina, Gasca, \& Camargo, 2019), la conservación en buen estado de sus activos (Komonen, Kortelainen, \& Räikkonen, 2006) y el aumento de la confiabilidad operacional (Arata, 2009), entre otras medidas.

El mantenimiento es la acción de restaurar los equipos a las condiciones para las cuales fue diseñado, esta práctica evita que los activos se degraden de forma anticipada, asegurando el mayor retorno de la inversión

\footnotetext{
${ }^{1}$ Dr. en Ciencias Mención: Gerencia. MSc. en Mantenimiento Industrial. Facultad de Ingeniería Mecánica, Electrónica y Biomédica. Universidad Antonio Nariño. Email: magasca@uan.edu.co

${ }^{2}$ Dr. en Ciencias Mención: Gerencia. MSc. en Ingeniería Electrónica. Facultad de Ingeniería, Universidad del Magdalena. Email:

Icamargoa@unimagdalena.edu.co

${ }^{3}$ Dr. en Ciencias Mención: Gerencia. MSc. en Ingeniería Electrónica. Facultad de Ingeniería, Universidad Francisco de Paula Santander. Email: byronmedina@ufps.edu.co
} 
(Amendola, 2013). La gestión del mantenimiento agrupa y aplica diferentes disciplinas, metodologías, buenas prácticas y herramientas del mantenimiento, con la intención de optimizar: el ciclo de vida de los equipos, los costos, el rendimiento, la exposición al riesgo, la disponibilidad, la confiabilidad, el cumplimiento de las regulaciones de seguridad y ambiente y, la armonía con el talento humano (García, 2012). Estos modelos de gestión deben ser integrales y deben alinearse con las estrategias, políticas e indicadores del negocio (Viveros, Stegmaier, Kristjanpoller, Barbera, \& Crespo, 2013).

La confiabilidad operacional está asociada con los procesos de mejora continua y con la productividad en las empresas, y se representa como la capacidad de una organización para desempeñar sus funciones de forma óptima en un lapso y bajo un entorno operacional específico (Holmberg \& Folkeson, 1991). Esta se puede determinar a partir de la confiabilidad del equipo, la confiabilidad humana, la confiabilidad del proceso y la mantenibilidad (Altmann, 2009).

A pesar de lo innovador y rentable que pueden resultar los conceptos de gestión del mantenimiento y la confiabilidad operacional, estos han sido poco aplicados en el sector de la industria manufacturera, especialmente en las pequeñas y medianas empresas (pymes), en el caso colombiano. Aunque este sector productivo sea uno de los más significativos para el producto interno bruto en algunas ciudades del país.

El informe económico de la Cámara de Comercio de la ciudad de Santa Marta de 2017, indica que en la ciudad se concentra el 68,7 \% de las unidades empresariales del departamento del Magdalena, equivalente a 19.612 empresas. El sector de industria manufacturera, durante el 2016 concentró el 16,5 \% de los activos, el 20,8 \% de las ventas y las 200 empresas más generadoras de desarrollo del departamento. Adicionalmente, informa que hay registradas 598 pymes en Santa Marta para diciembre del 2016 y aproximadamente el 5 \% de estas pertenecen a la industria manufacturera (Cámara de Comercio de Santa Marta para el Magdalena, 2017).

En el 2019 se registraron en el departamento del Magdalena 29.627 empresas, con el 96,09 \% micro, el 2,69 \% pequeñas, 0,92 \% medianas y el 0,3 \% grandes; además, los sectores con el mayor número de empresas fueron: comercio, alojamiento y servicios de comida, recreación y la industria manufacturera. En la ciudad de Santa Marta se ubicaron 5.050 nuevas matrículas mercantiles y 14.713 renovaciones. El sector industria manufacturera aporta el 6,4 \% de las nuevas empresas creadas con activos por 5.357 millones de pesos colombianos, y el 6,3 \% de las empresas que renovaron la matrícula mercantil con concentración de activos por 1.185.952 millones de pesos colombianos (Cámara de Comercio de Santa Marta para el Magdalena, 2020).

Esta dinámica de crecimiento hace necesario la implementación de modelos que faciliten la buena gestión de los activos y de los procesos en estas empresas; debido a que la carencia de estos puede incidir en el rendimiento de los equipos, ocasionar afecciones y deterioro en los activos y, afectar negativamente la producción, repercutiendo en la competitividad de la industria (Gasca, Camargo, \& Medina, 2017).

Por lo anteriormente expuesto, se formula un modelo de gestión del mantenimiento para las pequeñas y medianas empresas de la industria manufacturera. El modelo propuesto tiene el sistema de variables de la tabla 1. 
Tabla 1

Sistematización de categoría y operacionalización de las variables del modelo propuesto

\begin{tabular}{|c|c|c|}
\hline Variable & Dimensión & Indicador \\
\hline \multirow{3}{*}{$\begin{array}{c}\text { Gestión del } \\
\text { mantenimiento }\end{array}$} & Situación actual & $\begin{array}{l}\text { Renovación } \\
\text { Mantenimiento }\end{array}$ \\
\hline & Equipos & $\begin{array}{l}\text { Equipos activos } \\
\text { Equipos pasivos }\end{array}$ \\
\hline & Procesos & $\begin{array}{l}\text { Identificación } \\
\text { Planificación } \\
\text { Ejecución } \\
\text { Operación } \\
\text { Plan de acción }\end{array}$ \\
\hline \multirow{3}{*}{$\begin{array}{c}\text { Confiabilidad } \\
\text { operacional }\end{array}$} & Prácticas & $\begin{array}{l}\text { Metodología estándar PAS } \\
55 \\
\text { Mantenimiento centrado en } \\
\text { confiabilidad } \\
\text { Mantenimiento basado en } \\
\text { riesgo }\end{array}$ \\
\hline & & $\begin{array}{l}\text { Mantenimiento productivo } \\
\text { total } \\
\text { Análisis causa raíz } \\
\text { Retorno de inversión }\end{array}$ \\
\hline & $\begin{array}{c}\text { Requerimientos } \\
\text { operacionales }\end{array}$ & $\begin{array}{l}\text { Recursos humanos } \\
\text { Sistemas de información } \\
\text { Equipos } \\
\text { Recursos financieros }\end{array}$ \\
\hline
\end{tabular}

Fuente: elaboración propia

\section{Metodología}

Para la elaboración del modelo se estudia la población objetivo a partir de la medición y descripción de las variables de investigación "Gestión del mantenimiento" y "Confiabilidad operacional", posteriormente a esta carecterización se plantean las diretrices para la gestión de forma gráfica en el modelo de gestión propuesto. Para esto se desarrollan las siguientes actividades: (i) se realiza una revisión crítica y sistemática de literatura para identificar las teorías y los conceptos utilizadas en el modelo; (ii) se desarrolla y aplica un instrumento para la recolección de los datos; (iii) se analiza en detalle la parte cuantitativa y los elementos cualitativos derivados de cada respuesta, por indicador y dimensión; y (iv) se formulan los lineamientos que tienen como objetivo solucionar las dificultades encontradas y potencializar las fortalezas halladas en el diagnóstico.

Se selecciona como población de estudio a las treinta pymes, del sector manufacturero, ubicadas en el distrito de Santa Marta del Departamento del Magdalena, según registro de la Cámara de Comercio de Santa Marta y de acuerdo con el decreto 957 de 2019 del Ministerio de Comercio, Industria y Turismo de Colombia (MinCIT, 2019). Como la población de estudio es relativamente pequeña, esta es abarcada totalmente sin requerir un muestreo.

El instrumento aplicado a estas treinta pymes es un cuestionario tipo encuesta conformado por cincuenta y siete preguntas cerradas, con cinco alternativas de respuesta tipo escala de Likert, clasificadas como: siempre (con puntuación de 5), casi siempre (con puntuación de 4), a veces (con puntuación de 3), casi nunca (con puntuación de 2) y nunca (con puntuación de 1). El instrumento se valida con ayuda de expertos académicos en el área de la investigación, quienes evaluaron: la consistencia de las preguntas con los postulados teóricos; la redacción 
precisa, clara y sin ambigüedades de las mismas; y la correcta secuencia de estas. Para verificar la fiabilidad del instrumento, éste se aplicó a cinco profesionales expertos en la gestión del mantenimiento que no formaron parte de la población, posteriormente se utiliza la técnica de Alfa de Cronbach siguiendo la ecuación (1),

$$
r_{t t}=\frac{K}{K-1}\left[1-\frac{\sum S_{i}^{2}}{S_{t}^{2}}\right]
$$

dónde: $r t$, es coeficiente de Alfa de Cronbach, $K$ es el número de preguntas, $S_{i}$ es la varianza de las respuestas en cada pregunta, $S_{t}$ es la varianza de los totales. El valor obtenido de $r t t$ es de 0,87 permitiendo considerar al instrumento como confiable.

Para la interpretación de los resultados cuantitativos se construye el baremo que se muestra en la tabla 2. El intervalo para lo grupos $(0,8)$ se determina a partir de la diferencia entre el límite superior $(5)$ y el límite inferior (1), dividido en el número de atributos (5) del baremo.

Tabla 2

Baremo para la interpretación de la media

\begin{tabular}{ccc}
\hline Grupo & Rango de valores & Atributo \\
\hline 1 & $1 \leq R<1,8$ & Muy deficiente \\
2 & $1,8 \leq R<2,6$ & Deficiente \\
3 & $2,6 \leq R<3,4$ & Regular \\
4 & $3,4 \leq R<4,2$ & Bueno \\
5 & $4,2 \leq R \leq 5,0$ & Muy bueno \\
\hline
\end{tabular}

Fuente: elaboración propia

\section{Resultados}

Se realiza el análisis estadístico descriptivo de cada indicador y se contrastan los resultados con los referentes teóricos, con el propósito de proponer los lineamientos del modelo de gestión del mantenimiento para la confiabilidad operacional en pequeñas y medianas empresas de la ciuda de Santa Marta.

\subsection{Análisis descriptivo de los resultados}

Se interpretaron las respuestas de los 57 ítems, relacionados con 19 indicadores, 5 dimensiones y 2 variables.

Los resultados de la variable gestión del mantenimiento, se muestran en la tabla 3 y la figura 1. La tabla 4 relaciona las fortalezas y debilidades encontradas en las empresas en función de esta varible. 
Tabla 3

Evaluación de la variable gestión del mantenimiento

\begin{tabular}{|c|c|c|c|c|c|c|c|c|}
\hline \multicolumn{3}{|c|}{ Indicador } & \multicolumn{3}{|c|}{ Dimensión } & \multicolumn{3}{|c|}{ Variable } \\
\hline Nombre & $\bar{X}$ & Atributo & Nombre & $\bar{X}$ & Atributo & Nombre & $\bar{X}$ & Atributo \\
\hline Renovación & 3,28 & Regular & \multirow{2}{*}{$\begin{array}{c}\text { Situación } \\
\text { actual }\end{array}$} & \multirow{2}{*}{3,71} & \multirow{2}{*}{ Bueno } & \multirow{9}{*}{$\begin{array}{c}\text { Gestión del } \\
\text { mantenimiento }\end{array}$} & \multirow{9}{*}{3,68} & \multirow{9}{*}{ Bueno } \\
\hline Utilización & 4,14 & Bueno & & & & & & \\
\hline Equipos activos & 3,39 & Regular & \multirow{2}{*}{ Equipos } & \multirow{2}{*}{3,65} & \multirow{2}{*}{ Bueno } & & & \\
\hline Equipos pasivos & 3,92 & Bueno & & & & & & \\
\hline Identificación & 3,97 & Bueno & \multirow{5}{*}{ Procesos } & \multirow{5}{*}{3,68} & \multirow{5}{*}{ Bueno } & & & \\
\hline Planificación & 3,33 & Regular & & & & & & \\
\hline Ejecución & 4,22 & Muy Bueno & & & & & & \\
\hline Operación & 3,67 & Bueno & & & & & & \\
\hline Plan de acción & 3,19 & Regular & & & & & & \\
\hline
\end{tabular}

Fuente: elaboración propia

Figura 1

Representación de indicadores de la gestión del mantenimiento

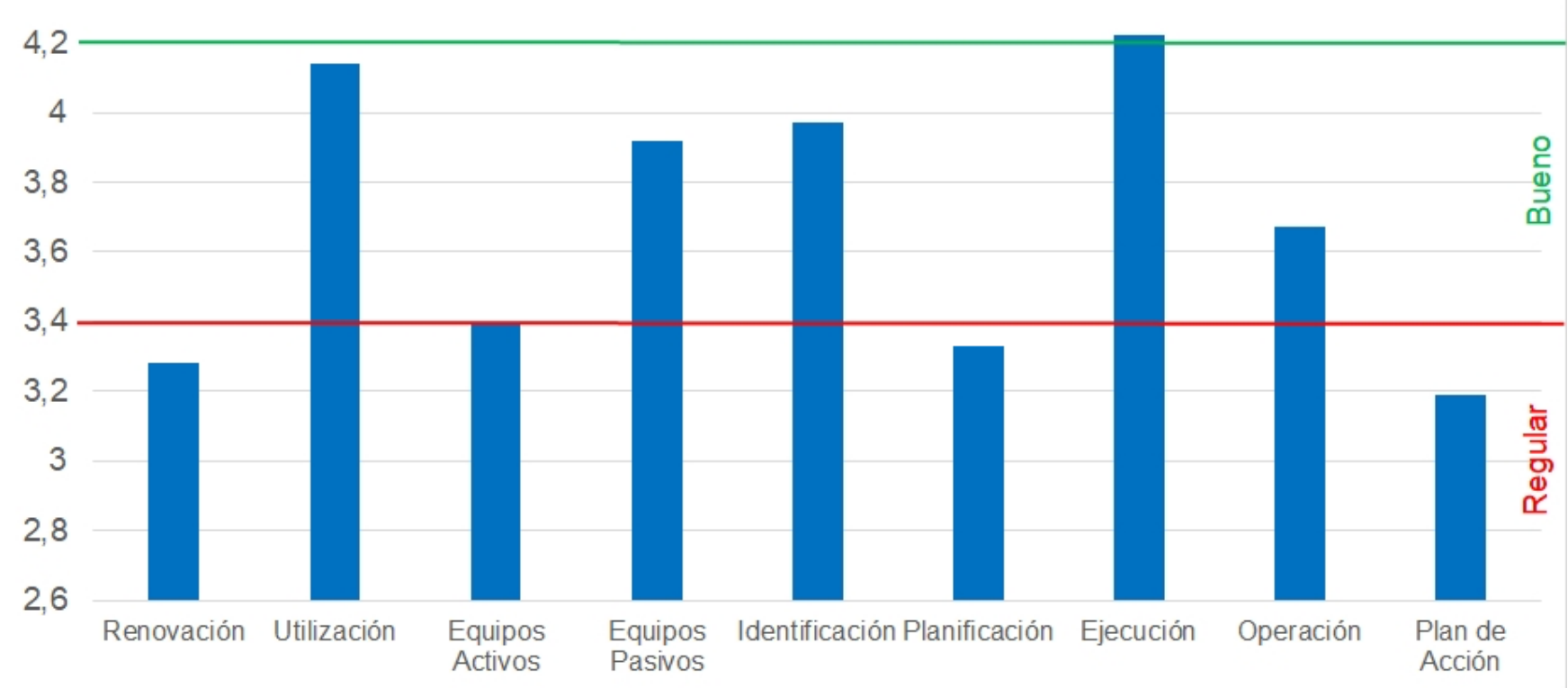

Fuente: elaboración propia 
Tabla 4

Hallazgos en la variable gestión del mantenimiento

\begin{tabular}{|c|c|c|c|}
\hline Dimensión & Indicador & Fortalezas & Debilidades \\
\hline \multirow[t]{2}{*}{ Situación actual } & Renovación & & $\begin{array}{l}\text { Regularmente remplazan equipos para aumentar la } \\
\text { productividad. } \\
\text { Regularmente se promueve el cambio de equipos con } \\
\text { base en la competitividad. } \\
\text { Moderadamente fomentan en los empleados el uso de } \\
\text { nuevos equipos. }\end{array}$ \\
\hline & Utilización & $\begin{array}{l}\text { Lideran las funciones operacionales. } \\
\text { Orientan las actividades y procesos operativos } \\
\text { de forma continua. } \\
\text { Utilizan los equipos de forma continua. }\end{array}$ & $\begin{array}{l}\text { Es prioritaria la gestión del mantenimiento que garantice } \\
\text { la confiabilidad operacional. }\end{array}$ \\
\hline \multirow[t]{2}{*}{ Equipos } & Equipos activos & $\begin{array}{l}\text { Procuran evaluar conjuntamente con el } \\
\text { personal la factibilidad de los equipos. } \\
\text { Realizan el seguimiento a los equipos nuevos. }\end{array}$ & $\begin{array}{l}\text { Los equipos se envejecen y se incrementan las fallas. } \\
\text { Esto aumenta los costos de mantenimiento, baja el } \\
\text { desempeño de los equipos y ocasiona gastos adicionales. } \\
\text { No procuran recuperar la inversión inicial, con la venta } \\
\text { de los equipos usados. }\end{array}$ \\
\hline & Equipos pasivos & $\begin{array}{l}\text { Eligen los equipos considerando los proyectos } \\
\text { y el servicio requerido por el cliente. }\end{array}$ & $\begin{array}{l}\text { Algunas veces no jerarquizan los equipos según el estado } \\
\text { operativo. }\end{array}$ \\
\hline \multirow{5}{*}{ Procesos } & Identificación & $\begin{array}{l}\text { Ordenan los procesos. } \\
\text { Divulgan las nuevas prácticas realcionadas con } \\
\text { los procesos. }\end{array}$ & No se describen los procesos por completo. \\
\hline & Planificación & $\begin{array}{l}\text { Procuran llevar la logística de funcionamiento } \\
\text { de los equipos. }\end{array}$ & $\begin{array}{l}\text { No siempre se cuenta con sistemas de información para } \\
\text { la planificación. } \\
\text { No todas las empresas evalúan las etapas del uso de los } \\
\text { equipos. }\end{array}$ \\
\hline & Ejecución & $\begin{array}{l}\text { Establecen objetivos anuales. } \\
\text { Formulan y evalúan políticas. } \\
\text { Motivan en los empleados la cultura de } \\
\text { estructura organizacional eficaz. }\end{array}$ & \\
\hline & Operación & $\begin{array}{l}\text { En la operación desarrollan estrategias } \\
\text { basadas en los proyectos. } \\
\text { Condicionan la operatividad de los equipos a } \\
\text { los cambios estratégicos. }\end{array}$ & $\begin{array}{l}\text { Regularmente consultan a su personal para la } \\
\text { adquisición de equipos. }\end{array}$ \\
\hline & Plan de acción & Priorizan la evaluación del riesgo. & $\begin{array}{l}\text { Pocas veces tienen planes para detectar fallas en los } \\
\text { equipos. } \\
\text { Regularmente evalúan la operación continua de los } \\
\text { equipos. }\end{array}$ \\
\hline
\end{tabular}

Fuente: elaboración propia

Los resultados de la confiabilidad operacional se muestran en la tabla 5 y en la figura 2. La tabla 6 relaciona las fortalezas y debilidades de la variable confiabilidad operacional 
Tabla 5

Evaluación de la variable confiabilidad operacional

\begin{tabular}{|c|c|c|c|c|c|c|c|c|}
\hline \multicolumn{3}{|l|}{ Indicador } & \multicolumn{3}{|c|}{ Dimensión } & \multicolumn{3}{|c|}{ Variable } \\
\hline Nombre & $\overline{\boldsymbol{X}}$ & Atributo & Nombre & $\overline{\boldsymbol{X}}$ & Atributo & Nombre & $\bar{X}$ & Atributo \\
\hline Metodología PAS 55 & 1,76 & Muy Deficiente & \multirow{6}{*}{ Prácticas } & \multirow{6}{*}{2,97} & \multirow{6}{*}{ Regular } & \multirow{10}{*}{$\begin{array}{c}\text { Confiabilidad } \\
\text { operacional }\end{array}$} & \multirow{10}{*}{3,38} & \multirow{10}{*}{ Regular } \\
\hline Mantenimiento centrado en confiabilidad & 3,38 & Regular & & & & & & \\
\hline Mantenimiento basado en riesgo & 3,29 & Regular & & & & & & \\
\hline Mantenimiento productivo total & 2,69 & Regular & & & & & & \\
\hline Análisis causa raíz & 2,9 & Regular & & & & & & \\
\hline Retorno de inversión & 3,81 & Bueno & & & & & & \\
\hline Recursos humanos & 4,22 & Muy Bueno & \multirow{4}{*}{$\begin{array}{c}\text { Requerimientos } \\
\text { operacionales }\end{array}$} & \multirow{4}{*}{3,78} & \multirow{4}{*}{ Bueno } & & & \\
\hline Sistema de información & 2,93 & Regular & & & & & & \\
\hline Equipos & 3,32 & Regular & & & & & & \\
\hline Recursos financieros & 4,67 & Muy Bueno & & & & & & \\
\hline
\end{tabular}

Fuente: elaboración propia

Figura 2

Representación de indicadores de la confiabilidad operacional

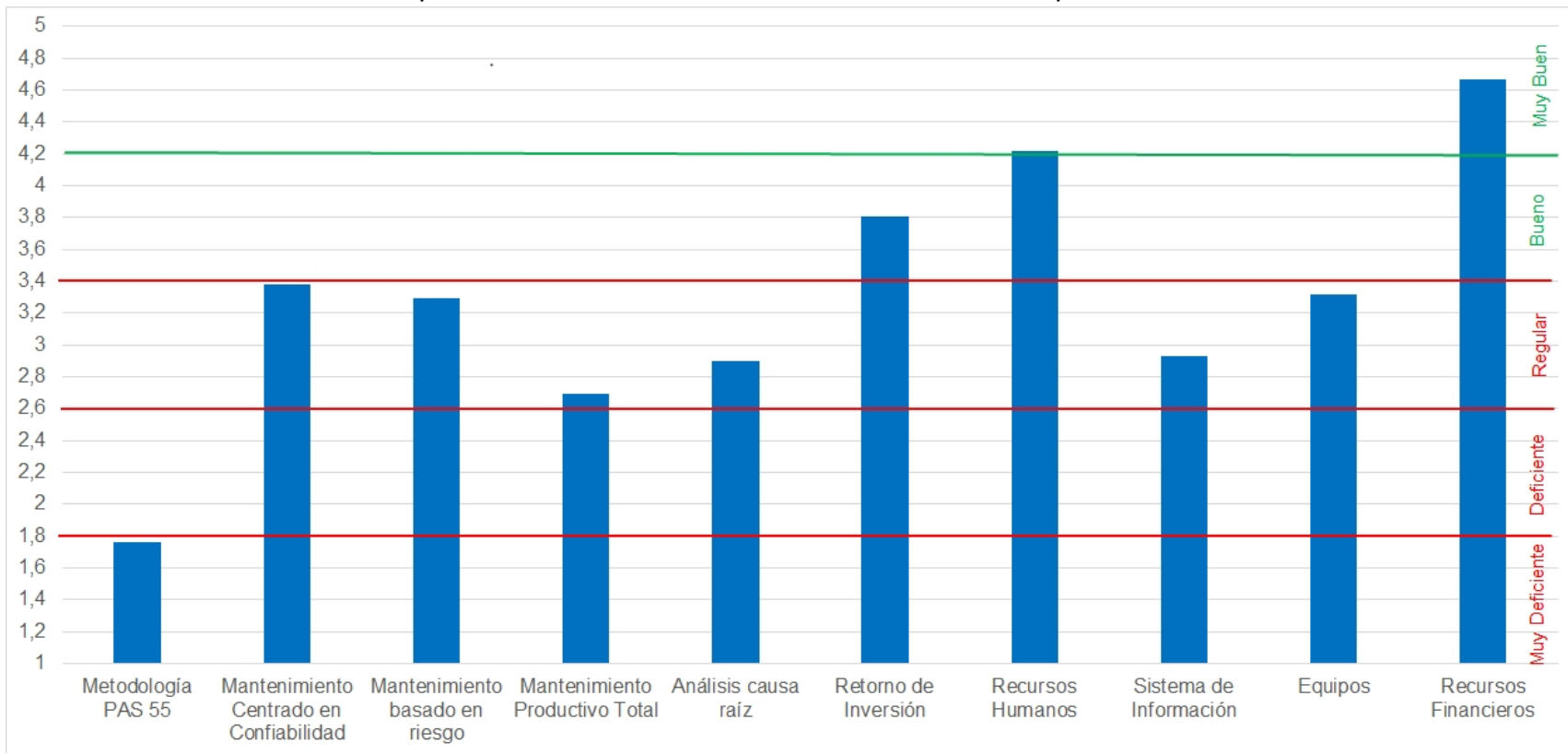

Fuente: elaboración propia 
Tabla 6

Hallazgos en la variable confiabilidad operacional

\begin{tabular}{|c|c|c|c|}
\hline Dimensión & Indicador & Fortalezas & Debilidades \\
\hline \multirow{6}{*}{ Prácticas } & Metodología PAS 55 & & $\begin{array}{l}\text { No conocen, ni aplican el concepto de esta práctica. } \\
\text { Regularmente consideran los costos para aplicar un } \\
\text { modelo organizativo de equipos. }\end{array}$ \\
\hline & $\begin{array}{l}\text { Mantenimiento } \\
\text { centrado en } \\
\text { confiabilidad }\end{array}$ & $\begin{array}{l}\text { Interés por estudiar las características de } \\
\text { las fallas en los equipos. }\end{array}$ & $\begin{array}{l}\text { Regularmente predicen las fallas de los equipos. } \\
\text { Pocas veces analizan las fallas de manera } \\
\text { multidisciplinaria. }\end{array}$ \\
\hline & $\begin{array}{l}\text { Mantenimiento } \\
\text { basado en riesgo }\end{array}$ & $\begin{array}{l}\text { Normalmente analizan las pérdidas antes } \\
\text { de invertir. }\end{array}$ & $\begin{array}{l}\text { Regularmente fijan estrategias para prevenir el riesgo. } \\
\text { Pocas veces estudian globalmente las pérdidas en el } \\
\text { contexto operacional. }\end{array}$ \\
\hline & $\begin{array}{l}\text { Mantenimiento } \\
\text { productivo total }\end{array}$ & & $\begin{array}{l}\text { Pocas veces participa el personal de producción en tareas } \\
\text { de mantenimiento. } \\
\text { Pocas veces consideran en conjunto los criterios de los } \\
\text { mantenedores y operadores, para reparar equipos. } \\
\text { Regularmente realizan mantenimientos preventivos. }\end{array}$ \\
\hline & Análisis causa raíz & & $\begin{array}{l}\text { Aceptable uso de herramientas de análisis causa raíz. } \\
\text { Moderadamente identifican las causas lógicas de las fallas. } \\
\text { Regular inversión de dinero para identificar las causas } \\
\text { lógicas de las fallas. }\end{array}$ \\
\hline & Retorno de inversión & $\begin{array}{l}\text { Establecen objetivos empresariales. } \\
\text { Las inversiones se realizan con base en el } \\
\text { retorno de la misma. }\end{array}$ & $\begin{array}{l}\text { Normalmente no se registra el control de los equipos con } \\
\text { herramientas electrónicas. }\end{array}$ \\
\hline \multirow{4}{*}{$\begin{array}{l}\text { Requerimientos } \\
\text { operacionales }\end{array}$} & Recursos humanos & $\begin{array}{l}\text { A menudo establecen responsabilidades y } \\
\text { respetan la jerarquía organizacional. } \\
\text { La adecuada asignación de roles al } \\
\text { personal, favorece la gestión del } \\
\text { mantenimiento. }\end{array}$ & \\
\hline & $\begin{array}{l}\text { Sistema de } \\
\text { información }\end{array}$ & & $\begin{array}{l}\text { Mayormente los sistemas de información están dirigidos a } \\
\text { la gerencia. } \\
\text { Dificultades de comunicación, entre la gerencia y el resto } \\
\text { de la organización, para la toma de decisiones. }\end{array}$ \\
\hline & Equipos & $\begin{array}{l}\text { Propician la utilización de equipos para } \\
\text { facilitar los trabajos. }\end{array}$ & $\begin{array}{l}\text { Algunas veces no usan adecuadamente los equipos. } \\
\text { Algunas veces no toman las medidas preventivas en el uso } \\
\text { de los equipos. }\end{array}$ \\
\hline & Recursos financieros & $\begin{array}{l}\text { Uso adecuado de los recursos financieros. } \\
\text { Asignación de recursos financieros con } \\
\text { base en los propósitos organizacionales. } \\
\text { Control financiero con balances que } \\
\text { evidencian la realidad económica. }\end{array}$ & \\
\hline
\end{tabular}

Fuente: elaboración propia

\subsection{Modelo propuesto}

Teniendo en cuenta las fortalezas y debilidades de las empresas y los fundamentos teóricos, se elaboró un modelo de gestión del mantenimiento para la confiabilidad operacional en las pequeñas y medianas empresas de la ciudad de Santa Marta. En la figura 3 se muestra el esquema conceptual del modelo. 
Figura 3

Modelo de gestión del mantenimiento para la confiabilidad operacional

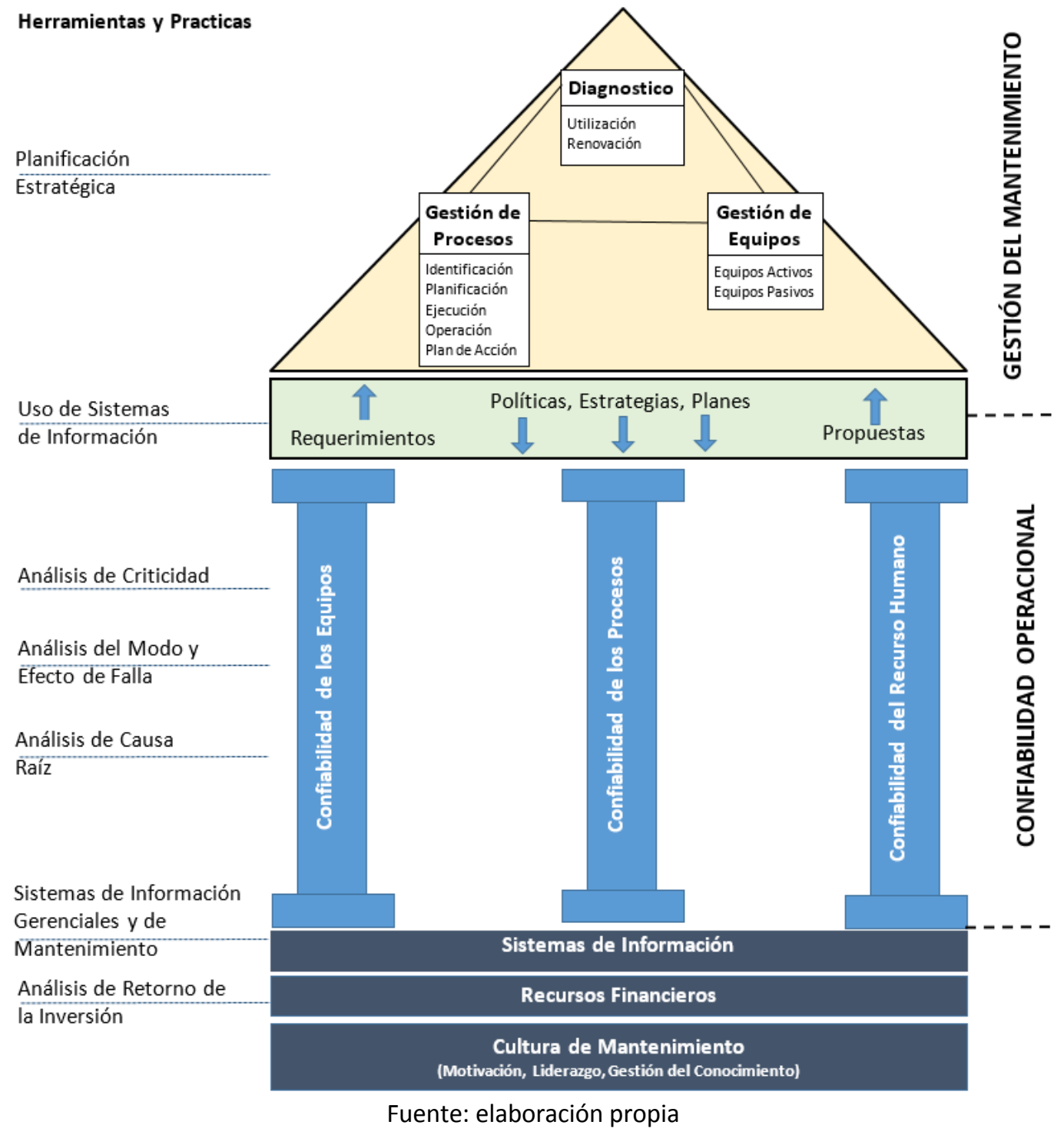

El esquema conceptual del modelo de gestión del mantenimiento para la confiabilidad operacional es análogo a una estructura de edificación que tiene cimientos, columnas, vigas y cubierta.

\section{Cimientos}

Los cimientos son la parte de una construcción que está bajo tierra y que da solidez, sirven de base, a la edificación. En el modelo los cimientos están representados por la cultura de mantenimiento, los recursos financieros y los sistemas de información.

La cultura de mantenimiento contempla la actitud, la motivación y las competencias del personal con respecto a las tareas de operación y mantenimiento de la empresa. Para generar esta cultura hay que desarrollar en los empleados habilidades de motivación y liderazgo; además de gestionar el conocimiento dentro de la empresa.

Los recursos financieros se refiere al capital disponible para invertir, cuando se requiera, en actividades de mantenimiento. Para asegurar los recursos financieros, se sugiere utilizar la métrica retorno de la inversión y establecer políticas de venta de equipos usados para dinamizar la renovación de éstos. 
Los sistemas de información son herramientas que monitorizan el estado de las variables de determinada área de la empresa, a partir de información interna y externa de la misma, para optimizar las actividades de mantenimiento. Los sistemas de información deben contener y relacionar información gerencial y de mantenimiento. La información de mantenimiento hace referencia a la taxonomía y logística de los equipos y, a los instrumentos de mantenimiento (rutinas de inspección, planes preventivos, recolección de datos, indicadores, e instrucciones).

\section{Columnas}

Las columnas son los elementos estructurales verticales de una edificación, en el modelo éstas tienen una relación directa con la variable de confiabilidad operacional y, representan la capacidad de la empresa para cumplir su propósito, en un contexto operacional específico.

El modelo tiene tres columnas llamadas confiabilidad de los equipos, confiabilidad de los procesos y confiabilidad del recurso humano; (i) la confiabilidad de los equipos garantiza la disponibilidad de éstos en las condiciones de operación para las que fue construido; (ii) la confiabilidad de los procesos está asociada con la comprensión y mejora de los procesos y procedimientos de la organización; y (iii) la confiabilidad del recurso humano es la probabilidad del rendimiento eficiente y eficaz del personal, en todos los procesos, manteniendo la integridad del sistema.

Las columnas deben ser contruidas con herramientas, técnicas y buenas prácticas para el mantenimiento como: análisis de criticidad, análisis de modos y efectos de fallas, análisis de causa raíz y, la realización de manuales de procedimientos.

El análisis de criticidad para jerarquizar áreas, equipos o partes de equipos, mediante la cuantificación de su impacto sobre las operaciones de la empresa, facilita la asignación de recursos y la definición de prioridades de trabajo. Este análisis establece los criterios a evaluar (seguridad industrial, ambiente, tiempo medio entre falla, costos de mantenimiento, costos operacionales, tiempo promedio de reparación y frecuencia de la falla); y define el método a utilizar (frecuencia por consecuencia, consecuencia por complejidad o impacto global).

El análisis de modos y efectos de fallas define la función real de los equipos, las fallas funcionales, los modos de fallas y los efectos de las fallas, para determinar las actividades de mantenimiento adecuadas, que evitan o disminuyan las consecuencias de las fallas. Este análisis se realiza de acuerdo con la norma SAE JA1012, es decir con base en las preguntas: ¿Cuál es la función del activo? ¿De qué manear puede fallar el activo? ¿Qué origina la falla en el activo? ¿Qué pasa cuando falla el activo? ¿lmporta si falla el activo? ¿Se puede hacer algo para prevenir la falla? ¿Qué pasa si no podemos prevenir la falla? (Society of Automotive Engineers, 2011).

El análisis de causa raíz se realiza cuando el equipo que presenta la falla es crítico y, la falla es crónica y recurrente, con el propósito de conseguir las causas raíces físicas y latentes del problema y, reducir inmediatamente el riesgo de ocurrencia de eventos mayores. Este análisis utiliza un árbol lógico que representa las relaciones de causa y efecto, para descubrir el evento indeseable y la causa raíz del problema. El procedimiento para hacer el análisis implica definir el evento de la falla, describir los modos de la falla, plantear las hipótesis de las causas de la falla, verificar las hipótesis a partir de las raíces físicas y humanas y, determinar las raíces latentes y verificables.

Los manuales de procedimiento de las tareas rutinarias de operación y mantenimiento, contribuyen a disminuir los errores humanos, evitar el riesgo y aumentar la confiabilidad operacional de la empresa. Estos manuales deben elaborarse con el personal que realiza las actividades de operación y mantenimiento, y utilizar como fundamento los resultados del análisis de modos y efectos de fallas.

\section{Vigas}


Las vigas son los elementos horizontales en una edificación, que soportan y transmiten las cargas transversales hacia las columnas. En el modelo, la viga transmite los requerimientos y propuestas de la variable confiabilidad operacional hacia la variable gestión del mantenimiento. Asimismo, transmite las políticas y estrategias de la variable gestión del mantenimiento hacia la variable confiabilidad operacional.

\section{Cubierta}

La cubierta es el elemento arquitectónico que está encima de otra para cubrirla. En el modelo, la cubierta simboliza la variable gestión del mantenimiento, compuesta por el diagnóstico, la gestión de procesos y la gestión de equipos, relacionados entre sí. El diagnóstico identifica la situación actual de la empresa en materia de mantenimiento, partiendo de los indicadores de utilización y renovación de los equipos. La gestión de procesos, hace referencia a las acciones de identificación, planificación, ejecución, operación y plan de acción, de los procesos. La gestión de equipos, activos y pasivos, optimiza este recurso, aumentando la productividad y competitividad de la empresa.

\section{Conclusiones}

Es posible desarrollar e implementar en las pequeñas y medianas empresas del sector de la industria manufacturera de la ciudad de Santa Marta un modelo de gestión del mantenimiento lógico y flexible, que combine las prácticas y las herramientas del mantenimiento centrado en confiabilidad y el mantenimiento productivo total; para el mejoramiento de la confiabilidad operacional.

Las pequeñas y medianas industrias manufactureras analizadas, normalmente despliegan actividades para garantizar que los equipos y los procesos operen de forma continua; pero pocas veces se formaliza y modela la gestión del mantenimiento en su funcionamiento interno. Adicionalmente, se percibe que estas empresas regularmente no reemplazan sus equipos de produción para aumentar la productividad y competitividad, y casi ninguna diseña planes de venta de equipos usados para facilitar la reinversión en tecnología.

Las actividades de mantenimiento en las empresas estudiadas normalmente no tienen en cuenta los sistemas de información para su planificación, generando errores y demoras; regularmente solo se consideran los criterios de los mantenedores y operadores. Tambien se observa que en las actividades es prioridad la evaluación del riesgo, lo que contrasta con el hecho de que la mayoría de estas industrias no cuenta con planes para detectar fallas en sus equipos, ni evalúan periódicamente el estado y ciclo de vida de los equipos. Teniendo en cuenta lo anterior, se recomienda a las empresas realizar con diligencia una descripción completa de los procesos, incluyendo los manuales de procedimiento; adquirir sistemas de información que faciliten la planificación del mantenimiento; y realizar revisiones semestrales a los equipos con tecnologías de diagnóstico que permitan predecir fallas, apoyando de esta manera los mantenimientos preventivos.

El personal de mantenimiento en las empresas encuestadas tiene conocimiento sobre las diferentes metodologías de mantenimiento, pero tan solo a veces éstas son aplicadas en la organización y en muchas ocasiones la aplicación se limita al uso de herramientas únicas asociadas a simple moda empresarial; esto lleva a que no se alcancen los resultados y metas esperadas por la gerencia. Estas empresas normalmente no promueven el uso de las diferentes metodologías de optimización dentro de un proceso global de mejora de la confiabilidad operacional en toda la organización.

Con respecto a los requerimientos operacionales las empresas: tienen claro los objetivos organizacionales y sustentan sus proyectos con base en el retorno de la inversión; analizan las pérdidas antes de invertir; realizan un control financiero con balances que evidencian la realidad económica; asignan los recursos financieros en función del propósito organizacional y; favorecen la confiabilidad operacional con la apropiada asignación de roles y responsabilidades del personal. Sin embargo, existen debilidades en la toma de decisiones por las

dificultades de comunicación entre la gerencia y el resto de la organización y; los sistemas de información son exclusivos de la gerencia y excluyen aspectos de la gestión del mantenimiento. 


\section{Referencias bibliográficas}

Altmann, C. (2009). ¿Cómo mejorar la confiabilidad de un sistema complejo? Revista Protección y Seguridad, 55(325), 59-68.

Amendola, L. (2013). Gestión integral del mantenimiento de activos como estrategia de negocios: (Assessment, PAS 55-ISO 55000). Mantenimiento: ingeniería industrial y de edificios(267), 12-19.

Arata, A. (2009). Ingeniería y gestión de la confiabilidad operacional en plantas industriales. Santiago de Chile: RIL Editores.

Cámara de Comercio de Santa Marta para el Magdalena. (2017). Informe Económico de 2016 de la Jurisdicción CCSM. Santa Marta: Centro de desarrollo empresarial. Área de estudios económicos. Recuperado de https://www.ccsm.org.co/servicios-empresariales/servicios-ofertas-empresariales/estudioseconomicos/investigaciones-e-indicadores-economicos/send/78-investigaciones/45652-informeeconomico-de-la-jurisdiccion-2016.html

Cámara de Comercio de Santa Marta para el Magdalena. (2020). Estructura empresarial del Magdalena. Santa Marta: Centro de desarrollo empresarial. Área de estudios económicos. Recuperado de https://www.ccsm.org.co/servicios-empresariales/servicios-ofertas-empresariales/estudioseconomicos/investigaciones-e-indicadores-economicos/send/78-investigaciones/49742-estructuraempresarial-del-magdalena-2019.html

García, O. (2012). Gestión Moderna del Mantenimiento Industrial. Principios fundamentales. Bogotá: Ediciones de la U.

Gasca, M., Camargo, L., y Medina, B. (2017). Sistema para evaluar la confiabilidad de equipos críticos en el sector industrial. Información tecnológica, 28(4), 111-124.

González, F. (2010). Auditoría del mantenimiento e indicadores de gestión (2 ed.). Madrid, España: FC Editorial. Fundación Confemetal.

Holmberg, K., y Folkeson, A. (1991). Operational reliability and systematic maintenance. New York: Elsevier.

Komonen, K., Kortelainen, H., y Räikkonen, M. (2006). An asset management framework to improve longer term returns on investments in the capital intensive industries. En J. Mathew, J. Kennedy, L. Ma, A. Tan, \& D. Anderson, Engineering asset management (págs. 418-432). Londres: Springer.

Medina, B., Gasca, M., y Camargo, L. (2019). Modelo de transferencia tecnológica para la competitividad de medianas empresas. Revista Espacios, 40(42), 18-33. Recuperado de https://www.revistaespacios.com/a19v40n42/a19v40n42p18.pdf

MinCIT. (5 de Junio de 2019). Decreto Número 957. Ley. Bogotá, Colombia: Ministro de Comercio, Industria y Turismo.

Rojas, M., Valencia, M., y Cuartas, D. (2017). Optimización racional de costos. Revista Espacios, 38(39), 8-19. Recuperado de https://www.revistaespacios.com/a17v38n39/a17v38n39p34.pdf

Society of Automotive Engineers. (22 de Agosto de 2011). SAE JA1012. A Guide to the reliability-centered maintenance (RCM) standard. SAE International.

Viveros, P., Stegmaier, R., Kristjanpoller, F., Barbera, L., y Crespo, A. (2013). Propuesta de un modelo de gestión de mantenimiento y sus principales herramientas de apoyo. Ingeniare. Revista chilena de ingeniería, 21(1), 125-138.

Esta obra está bajo una Licencia Creative Commons Attribución-NoCommercial 4.0 International

(cc) BY-NC 